\title{
A STUDY OF THE OPTICAL LONG-TERM BEHAVIOUR OF QUASARS
}

\author{
H. MEUSINGER, S. KLOSE, R. ZIENER \\ Thüringer Landessternwarte \\ Karl-Schwarzschild-Observatorium \\ D-07778 Tautenburg \\ Germany
}

\begin{abstract}
We report first results of a photometric investigation of the optical long-term behaviour of a sample of quasars. Our study is based on the plate archive of the Tautenburg $134 \mathrm{~cm} \mathrm{Schmidt} \mathrm{telescope}$ which covers a time baseline of over 30 years.
\end{abstract}

\section{Introduction}

Over the years compelling evidence has accumulated that variability is a common feature of quasars (Hawkins \& Veron 1993). Which fraction of quasars shows detectable optical variability is still a matter of debate. Recent estimates range from about $50 \%$ to nearly $100 \%$ (Cimatti et al. 1991).

The plate archive of the Tautenburg Schmidt telescope $\left(f=4 \mathrm{~m}, 3.3^{\circ} \times 3.3^{\circ}\right.$ vignetting-free field) covers a time baseline of about 30 years and is therefore well-suited to contribute to the study of the long-term behaviour of quasars.

\section{Quasars in the $M 3$ Field}

The field centred on $M 3$ belongs to the most intensively studied fields with the Tautenburg Schmidt. About 200 plates $(\approx 50 \%$ in $B$ ) were exposed during the years $1965-76$ and 1986 - 93. The questions we mainly addressed here were: (1) what is the fraction of variable quasars for such a long time baseline? (2) Is there a detectable long-term variability mode among these objects?

From published quasar catalogues we identified unambiguously 27 objects on a deep plate in an area about $1^{\circ}$ around $\mathrm{M} 3$ by measuring their relative coordinates with reference to SAO stars. Only measurements with secure identification of the quasars were used in the further data reduction. More than $90 \mathrm{~B}$-plates were measured using a semi-automated iris photometer. 30 plates were later rejected because of a relatively high error in the calibration curve. This often concerned plates exposed under good seeing conditions. Special attention was focused on the problem of a proper correction for irregularities in the plate background. The photometric reference stars were selected from the list of Johnson \& Sandage (1956).

We used the standard deviation $\sigma$ as a measure of the variability of an object and applied an 
$F$ test to check the hypothesis that the quasars show no variability. The 'instrumental' variance $\sigma_{\text {INST }}^{2}$ was estimated from the measured magnitudes of comparison stars in the quasar field assuming $\sigma_{I N S T}=\sigma_{\text {.. }}$. It shows a trend with $B$ and becomes more uncertain for $\langle B\rangle \geq 20^{\mathrm{m}}$. We therefore restricted the test to those 18 objects having $\left\langle B>\leq 19^{m} .5\right.$. For the quasars in the final sample the fraction of variables is estimated to be about 50 to $70 \%$. This result is confirmed by means of structure function analysis which is a robust method making allowances for poorly sampled data (Simonetti et al. 1985). Furthermore, the $t$ test indicates that most of the variable quasars have a long-term mode of variability.

Notable objects are Q $1340+289$ and $Q 1337+283$, the two brightest quasars in our sample. The former, with $\langle B\rangle=17.2, z=0.9$, is a structureless flat-spectrum radio quasar (Fanti et al. 1975). Comparison with published data from 40 to 50 years old Harvard plates (Mullikin \& Miller 1977) revealed a smooth dimming by about 2.5 mag. The remarkable feature of Q $1337+238(B=17.5, z=2.52)$ is its rather constant lightcurve, which shows no significant signs of variability.

\section{3. $Q 1803+676=\operatorname{Kaz} 102$}

Kazaryan 102 is a close-by radio-quiet quasar $\left(B \approx 16^{m}, z=0.138\right)$ with radio-loud properties (Evans II \& Natta 1989). Spectra of KAZ 102 obtained by Zheng et al. (1990) showed a variation in the continuum level at about $4400 \AA$ between August 1980 and September 1981 . Zheng et al. argued that this could be caused by a gas cloud in the AGN region crossing the line of sight.

The wavelength range where the spectral variation was observed is just at the centre of the Johnson $B$-passband. This motivated us to measure the magnitude of KAZ 102 on the $B$-plates of the north-ecliptic pole field which are available in the Tautenburg archive and which cover the timespan from 1979-81 and 1990-93. Photometric measurements were done using reference stars located within 5 arcmin around the quasar. We found that at the time of the observed spectral variability the quasar was about 0.4 mag fainter than in the year 1990 on average. Furthermore, evidence for a short-term variability mode was found.

\section{Acknowledgements}

This research has made use of the SIMBAD database, operated at CDS, Strasbourg, France. We are grateful to B. Stecklum, Jena, for computational support and C. Simon, Göttingen, for making available unpublished data of photometric reference stars.

\section{References}

Cimatti, A., Zamorani, G. and Marano, B., 1991. In Proc 'X-ray emission from active galactic nuclei and the cosmic X-ray background', eds. W. Brinkmann and J. Trümper, Garching, FRG.

Evans II, N.J. and Natta, A., 1989. Astrophys. J., 339, 943.

Fanti, C. et al., 1975. Astron. Astrophys. Suppl. Ser., 19, 143.

Hawkins, M.R.S. and Véron, P., 1993. Mon. Not. R. astron. Soc., 260, 202. 
Johnson, H.L. and Sandage, A., 1956. Astrophys. J., 124, 379.

Mullikin, T.L. and Miller, H.R., 1977., Publ. Astron. Soc. Pacific, 89, 639.

Simonetti, J.H., Cordes, J.M. and Heeschen, D.S., 1985. Astrophys. J., 296, 46.

Zheng, W., Burbidge, E.M. and Smith, H.E., 1990. Publ. Astron. Soc. Pacific, 102, 497. 